\title{
TRASLADO TRANSFRONTERIZO Y FIJACIÓN TEMPORAL DEL CRITERIO DE LA RESIDENCIA HABITUAL DEL MENOR COMO FORO DE COMPETENCIA JUDICIAL INTERNACIONAL: COMENTARIO AL AUTO DE LA AUDIENCIA PROVINCIAL DE CÓRDOBA DE 16 DE JULIO DE 2020
}

\author{
CROSS-BORDER MOVEMENT AND TEMPORARY FIXING \\ OF THE CRITERION OF HABITUAL RESIDENCE OF THE \\ CHILD AS A FORUM OF INTERNATIONAL JUDICIAL \\ COMPETENCE: COMMENTARY ON THE ORDER OF THE \\ PROVINCIAL COURT OF CÓRDOBA OF JULY 16, 2020
}

\author{
Laura Álvarez AgOuÉs \\ Investigadora Becaria predoctoral. Gobierno Vasco \\ Universidad del País Vasco. UPV/EHU
}

Recibido: 04.06.2021 / Aceptado: 21.06.2021

DOI: https://doi.org/10.20318/cdt.2021.6277

\begin{abstract}
Resumen: Esta resolución judicial, dictada en el marco de un litigio derivado de la reivindicación del ejercicio del derecho de visita planteada por la abuela paterna de los dos menores en un supuesto con elemento de internacionalidad, resuelve una cuestión recurrente en estos supuestos y concretada en la necesidad de analizar y fijar temporalmente el momento del cambio de residencia habitual sobrevenido de la madre y de sus hijos menores, que se trasladan desde España a Alemania con consentimiento del padre. Desde una dimensión procesal el Auto resuelve de forma correcta la determinación de qué tribunales son internacionalmente competentes para analizar la modificación de medidas solicitadas y vinculadas al ejercicio de derecho de visita al amparo del artículo 8 del Reglamento 2201/2003.

Palabras clave: traslado transfronterizo lícito, factor temporal, residencia habitual del menor, derecho de visita, competencia judicial internacional.

Abstract: This judicial resolution, issued in the framework of a dispute derived from the claim to exercise the right of visitation raised by the paternal grandmother of the two minors in a case with an internationality element, resolves a recurring issue in these cases and specified in the need to analyze and temporarily fix the time of the change of habitual residence of the mother and her two minor children, who move from Spain to Germany with the consent of the father. From a procedural dimension, the Order correctly resolves the determination of which courts are internationally competent to analyze the modification of measures requested and linked to the exercise of access rights under Article 8 of Regulation 2201/2003.
\end{abstract}

Keywords: lawful cross-border movement, time factor, habitual residence of the child, rights of access, international jurisdiction.

Sumario: I. Consideraciones introductorias. II. Decisiones judiciales susceptibles de modificación en Derecho de familia con factor de internacionalidad. III. Libertad de circulación y cam- 
bio sobrevenido de residencia habitual: el interés superior del menor. IV. Atribución competencial frente a distribución: competencia judicial internacional versus competencia territorial interna. V. Consideraciones finales.

\section{Consideraciones introductorias}

1. La resolución judicial objeto del presente comentario resuelve de forma acertada un debate procesal recurrente en la práctica litigiosa de crisis matrimoniales con componente de internacionalidad: por un lado, y el marco de una reclamación de modificación de medidas vinculada al ejercicio del Derecho de visita, se plantea una cuestión fáctica sobrevenida y concretada en el cambio de residencia habitual debido a la movilidad transfronteriza de la madre y de sus dos hijos menores, que se trasladan desde España a Alemania con consentimiento del padre (residente en Suiza).

2. Por otro, y desde una dimensión procesal, la decisión judicial analizada resuelve de forma correcta (aunque con una argumentación asistemática y poco diáfana) la concreción de qué tribunales son internacionalmente competentes, al amparo del artículo 8 del Reglamento ${ }^{\circ}$ 2201/2003 del Consejo, de 27 de noviembre de 2003, relativo a la competencia, el reconocimiento y la ejecución de resoluciones judiciales en materia matrimonial y de responsabilidad parental (en adelante, Reglamento Bruselas II bis) para acordar la modificación de medidas vinculadas al ejercicio de derecho de visita planteada por la abuela paterna de los dos menores.

4. La sentencia objeto de comentario plantea un problema recurrente en el marco del Derecho de familia internacional como es el derivado de la movilidad transfronteriza de las personas (y en particular, la de los menores) y el momento en el que, en relación a una cuestión litigiosa concreta, haya de precisarse el foro de competencia judicial internacional de la residencia habitual de éstos. Se añade a ello (y este caso es buena muestra de ello) y en atención a las materias abordadas en las mismas, el carácter no definitivo de ciertas sentencias (o de ciertas partes o pronunciamientos de las mismas) dictadas en materia de Derecho de familia.

5. ¿Qué debe entenderse por «resoluciones susceptibles de modificación? Tal y como acertadamente ha señalado A. Rodríguez Benot ${ }^{1}$, se trata de supuestos propios del Derecho de familia en que los cambios de las circunstancias fácticas sobre las que se basó la sentencia dictada por un tribunal de otro Estado resultan frecuentes: pensiones entre cónyuges, alimentos entre parientes, guarda y custodia de menores, protección de éstos o de personas con capacidad judicialmente modificada, entre otras.

\section{Decisiones judiciales susceptibles de modificación en Derecho de familia con factor de interna- cionalidad}

6. Cabría plantear de forma genérica las siguientes cuestiones, para proyectar su respuesta al caso ahora planteado: ¿se puede plantear la modificación de medidas ante otros tribunales de la Unión Europea distintos de los tribunales que dictaron la decisión en primer lugar?; ¿retiene su competencia judicial internacional el tribunal que dictó originariamente la sentencia?

7. En el ámbito de Derecho de familia, la competencia para instar el procedimiento de modificación de medidas no la retiene indefinidamente el juzgado que dictó en la originaria sentencia, la que ahora se solicita modificar. Es decir, dicho juzgado no conserva con carácter exclusivo la competencia de forma que, partiendo de la premisa de que el nuevo centro de vida se ubique en otro Estado de la

\footnotetext{
${ }^{1}$ A. Rodríguez Benot, "La Ley de cooperación jurídica internacional en materia civil", Cuadernos de Derecho Transnacional, marzo-2016, p.252.
} 
Unión Europea, los tribunales donde radique esa nueva residencia habitual podrán ser competentes para decretar la modificación de medidas mediante sentencia sobrevenida. En particular, y en relación a aquellas decisiones que afecten a menores, el artículo 9 del Reglamento Bruselas II bis regula bajo qué condiciones mantiene la competencia el Estado miembro de la anterior residencia habitual del menor ${ }^{2}$.

8. En efecto, y de conformidad con el art. 8.1 del Reglamento, la regla general en estos casos establece la competencia judicial internacional a favor de los órganos judiciales del Estado Miembro en cuyo territorio el menor tenga su residencia habitual. Sin embargo, si el menor traslada legalmente su residencia habitual de un Estado Miembro a otro, adquiriendo en este último su residencia habitual, entonces los órganos judiciales del Estado de la primera residencia habitual continuarán siendo los competentes durante los tres meses siguientes al cambio de residencia (art. 9).

9. ¿Qué sucede en el supuesto (planteado en el caso que da origen a la decisión ahora comentada) de que en el transcurso de un procedimiento judicial ya iniciado el menor cambie de residencia de manera legal? En tal supuesto, si el juez del Estado de la primera residencia habitual no se hubiese pronunciado aún, es decir, si no se hubiera dictado sentencia, dicho órgano judicial continuará reteniendo su competencia aunque el menor cambie su residencia en ese ínterin procesal. Y ello, siguiendo el principio general de perpetuatio fori. Este principio se aplica en el marco del Reglamento ${ }^{3}$.

10. El supuesto ahora planteado, más allá de la confusa descripción de los hechos probados en la sentencia, se concreta en tres circunstancias relevantes desde el punto de vista procesal: en primer lugar, es importante subrayar que el padre tiene su residencia habitual en Suiza y que la madre y los hijos menores bajo su custodia tienen su residencia en España en el momento del planteamiento de la litis; en segundo lugar, debe tenerse en cuenta que posteriormente, en pleno desarrollo del proceso, se trasladan a Alemania y fijan su nueva residencia habitual allí (traslado consentido por el padre) y por último, y en tercer lugar, debe atribuirse la competencia judicial internacional atendiendo a las previsiones del Reglamento 2201/2003; solo si, como sucede en el caso ahora resuelto, ésta recae sobre los tribunales españoles (competencia de atribución) podrá descenderse a la concreción de la competencia interna funcional y territorial (competencia de distribución). Ambos planos parecen haberse combinado incorrectamente en el actuar procesal de la parte actora, tal y como a continuación será analizado.

11. Se ha previsto expresamente la coexistencia de varios tribunales competentes, sin que entre ellos se haya establecido una jerarquía» (STJUE de 16-7-2009, asunto C-168/08, Hadadi, s.r., considerando 49). Para el TJUE los foros del art. 3 deben interpretarse de forma autónoma, sin atender al derecho de los distintos Estados miembros. En este caso, la solución al problema planteado pasa por la primacía del criterio de temporalidad, en el sentido de que los Tribunales en que se presente la demanda en primer lugar van a ser los competentes, debiéndose declarar la litispendencia al amparo de los artículos 19 del Reglamento (CE) No 2201/2003 y 12 del Reglamento (CE) No 4/2009.

12. Derivado de lo anterior, surge otra cuestión: si el artículo 8 del Reglamento Bruselas II bis y el artículo 3 del Reglamento 4/2009 deben interpretarse en el sentido de que los órganos jurisdiccionales

\footnotetext{
2 Reglamento 2201/2003, art. 9: “Cuando un menor cambie legalmente de residencia de un Estado miembro a otro y adquiera una nueva residencia habitual en este último, los órganos jurisdiccionales del Estado miembro de la anterior residencia habitual del menor seguirán siendo competentes, como excepción al artículo 8, durante los tres meses siguientes al cambio de residencia, para modificar una resolución judicial sobre el derecho de visita dictada en dicho Estado miembro antes de que el menor hubiera cambiado de residencia, si el titular del derecho de visita con arreglo a la resolución judicial sobre el derecho de visita continúa residiendo habitualmente en el Estado miembro de la anterior residencia habitual del menor. 2. El apartado 1 no se aplicará si el titular del derecho de visita considerado en el apartado 1 ha aceptado la competencia de los órganos jurisdiccionales del Estado miembro de la nueva residencia habitual del menor al participar en un procedimiento ante dichos órganos sin impugnar su competencia".

${ }^{3}$ Sobre el particular, vid. M. A. Aránzazu Gandía Sellens, "El Régimen Europeo de responsabilidad parental: su funcionamiento y las principales propuestas de cambio a raíz de la modificación del Reglamento Bruselas II bis", La Ley, Derecho de familia $\mathrm{n}^{\mathrm{o}}$ 17, enero-marzo 2018, Editorial Wolters Kluwer.
} 
de un Estado miembro que han adoptado una resolución firme en materia de responsabilidad parental y de obligaciones de alimentos en lo que respecta a un menor de edad siguen siendo competentes para conocer de una demanda de modificación de las medidas establecidas en esa resolución aunque la residencia habitual del menor esté situada en el territorio de otro Estado miembro 4 . Esto fue planteado como cuestión prejudicial por un tribunal de Lituania siendo resuelta por la STJUE de 15 de febrero de 2017, en el sentido de que dichos órganos jurisdiccionales no siguen siendo competentes para conocer de esa demanda de modificación de medidas sino que serán competentes los órganos jurisdiccionales del Estado miembro donde resida el menor.

13. Esto último implica que cualquier cambio sobrevenido en el devenir de ese litigio (por ejemplo, un traslado consentido a Alemania como en el supuesto recogido en la decisión objeto de este comentario) no afecta a la competencia judicial internacional de origen que recae sobre los tribunales españoles. Tiene tan solo una derivada procesal concreta: que si se notifica para emplazamiento a la madre como parta demandada, tal emplazamiento se dirigirá al nuevo lugar de residencia, que en el caso concreto sería Alemania. Brevemente, la competencia sobre el fondo no cambia, lo que cambia sería el acto de trámite (que es instrumental).

\section{Libertad de circulación y cambio sobrevenido de residencia habitual: el interés superior del menor}

14. La libertad de movimiento de las personas físicas, padres e hijos menores de edad, -en su consideración como derecho de la persona-, no viene determinada únicamente por el ordenamiento interno español sino también por el Derecho de la Unión europea. De esa manera, puede afirmarse, con F. Salerno, que la libertad de circulación adquiere una dimensión supranacional ${ }^{5}$. El derecho a la libre circulación de las personas se recoge en el art. 18 del Tratado Constitutivo de la Unión Europea ${ }^{6}$, así como también en el art. 45 de la Carta de los Derechos Fundamentales de la Unión Europea ${ }^{7}$. La Directiva 2004/38/CE del Parlamento Europeo y del Consejo, de 29 de abril de 2004, relativa al derecho de los ciudadanos de la Unión y de los miembros de sus familias ${ }^{8}$, pretende alentar a los ciudadanos de la Unión Europea a ejercer su derecho a la libre circulación y residencia en los Estados miembros ${ }^{9}$. Por

${ }^{4}$ B. UReÑa Carazo, "Competencia judicial internacional y modificación de medidas: pautas para su determinación", $L A$ $L E Y$, Derecho de familia ${ }^{\circ}$ 17, enero-marzo 2018, Normativa europea sobre Derecho de Familia, $\mathrm{N}^{\circ} 17,1$ de ene. de 2018, Editorial Wolters Kluwer.

5 F. SALERNo, "I diritti fondamentali della persona straniera nel diritto internazionale privato: un proposta metodologica", RDIPP, 2014, pp. 773-802. También, en el mismo sentido, R. BARATTA, "Derechos fundamentales y Derecho internacional privado de familia", AEDIP, t. XVI, 2016, pp. 103-126.

${ }^{6}$ Art. 21 (antiguo artículo 18 TCE) TFUE: "1. Todo ciudadano de la Unión tendrá derecho a circular y residir libremente en el territorio de los Estados miembros, con sujeción a las limitaciones y condiciones previstas en los Tratados y en las disposiciones adoptadas para su aplicación. | 2. Cuando una acción de la Unión resulte necesaria para alcanzar este objetivo, y a menos que los Tratados hayan previsto los poderes de acción al respecto, el Parlamento Europeo y el Consejo podrán adoptar, con arreglo al procedimiento legislativo ordinario disposiciones destinadas a facilitar el ejercicio de los derechos contemplados en el apartado 1.|3. A los efectos contemplados en el apartado 1, y salvo que los Tratados establezcan poderes de actuación para ello, el Consejo podrá adoptar, con arreglo a un procedimiento legislativo especial, medidas sobre seguridad social o protección social. El Consejo se pronunciará por unanimidad previa consulta al Parlamento Europeo".

7 Art. 45 de la Carta de los Derechos Fundamentales de la Unión Europea: "Todo ciudadano de la Unión tiene derecho a circular y residir libremente en el territorio de los Estados miembros. 2. De conformidad con lo dispuesto en el Tratado constitutivo de la Comunidad Europea, se podrá conceder libertad de circulación y de residencia a los nacionales de terceros países que residan legalmente en el territorio de un Estado miembro".

${ }^{8}$ Directiva 2004/38/CE del Parlamento Europeo y del Consejo, de 29 de abril de 2004, relativa al derecho de los ciudadanos de la Unión y de los miembros de sus familias a circular y residir libremente en el territorio de los Estados miembros por la que se modifica el Reglamento (CEE) n 1612/68 y se derogan las Directivas 64/221/CEE, 68/360/CEE, 72/194/CEE, 73/148/ CEE, 75/34/CEE, 75/35/CEE, 90/364/CEE, 90/365/CEE y 93/96/CEE (Texto pertinente a efectos del EEE) (DOUE L 158 de 30 abril 2004.

${ }^{9}$ En virtud de la Directiva 2004/38/CE, se consideran miembros de la familia a: el cónyuge (también cuando es del mismo sexo, tal como precisó el Tribunal de Justicia de la Unión Europea en su STJUE 5 junio 2018, C-673/16, Coman-Hamilton 
otra parte, la libertad de circulación y residencia ha de ponerse en relación con lo establecido en el art. 139.2 de la Constitución española, de manera que ninguna autoridad podrá adoptar medidas que directa o indirectamente obstaculicen la libertad de circulación y establecimiento de personas en todo el territorio español.

15. Cuando el progenitor cuidador principal, a quien se ha atribuido la guarda y custodia del hijo menor por resolución judicial, expresa su deseo de trasladar su domicilio está ejerciendo su derecho a la libre circulación. Ahora bien, si pretende trasladar también el domicilio del hijo menor, y puesto que dicha decisión no corresponde de forma unilateral al progenitor custodio, su derecho fundamental de residencia, domicilio y deambulación habrá de ser valorado en relación con el interés del menor ${ }^{10}$, que debe ser tutelado de manera preferente. Y al realizar ese juicio de valor, el Tribunal Supremo se ha pronunciado en el sentido de adoptar decisiones que tiendan a obtener el mayor beneficio para el menor en caso de traslado de su domicilio, de tal manera que en caso de incidir en el derecho del progenitor en su libertad de movimiento, prevalecerá el interés del menor incluso si ello pudiera comportar un cambio en el régimen de guarda y custodia acordado previamente mediante sentencia judicial. Así, la sentencia del Tribunal Supremo de 26 de octubre de $2012^{11}$ precisa:

"Es cierto que la Constitución Española en su artículo 19 determina el derecho de los españoles a elegir libremente su residencia, y a salir de España en los términos que la ley establezca. Pero el problema no es este. El problema se suscita sobre la procedencia o improcedencia de pasar la menor a residir en otro lugar, lo que puede comportar un cambio radical tanto de su entorno social como parental, con problemas de adaptación. De afectar el cambio de residencia a los intereses de la menor, que deben de ser preferentemente tutelados, podría conllevar, un cambio de la guarda y custodia".

16. La mencionada sentencia $n^{\circ} 642 / 2012$, del Tribunal Supremo, Sala $1^{\text {a }}$, de lo Civil, de 26 de octubre de 2012, constituye todo un referente de primera magnitud para fijar el régimen jurídico del cambio de domicilio del menor y para calificarlo como un verdadero y auténtico "acto de ejercicio extraordinario de la patria potestad". Esta línea es constante en la jurisprudencia del Tribunal Supremo ${ }^{12}$. Siguen el mismo criterio que la anterior las sentencias del Tribunal Supremo de 20 octubre 2014 y de 18 enero $2017^{13}$.

17. Una vez producida la ruptura del vínculo de pareja, ya sea por divorcio o por cese de la convivencia de la unión de hecho, no es extraño que uno de los miembros de la pareja desee iniciar junto con sus hijos menores una nueva vida en lugares distintos a los que se asentó inicialmente el núcleo familiar, bien por motivos de oportunidad laboral, bien por falta de arraigo o de vínculos culturales o sociales en el lugar del domicilio familiar, bien por la necesidad de cambiar de vida o incluso por haber formado una nueva familia con otra persona residente en una población distinta, o en un país diferente. Puede que se trate de un traslado de domicilio definitivo o que simplemente obedezca a situaciones de carácter temporal, como la realización de un proyecto laboral concreto, o la enfermedad de un familiar cercano que vive en otro lugar, o el cursar estudios por un periodo de tiempo determinado. La realidad es multiforme y supera toda imaginación.

[ECLI:EU:C:2018:385]), la pareja registrada, si la legislación del Estado miembro de acogida equipara la relación de pareja registrada al matrimonio; los descendientes directos menores de veintiún años o a cargo, incluidos los del cónyuge o de la pareja registrada; y los ascendientes directos a cargo, incluidos los del cónyuge o de la pareja registrada.

10 S. Álvarez González, "Responsabilidad parental, transferencia de la competencia a los órganos jurisdiccionales de otro Estado miembro e interés superior del menor", LA LEY Unión Europea no 43, diciembre 2016, n 43, 30 de dic. de 2016, Editorial Wolters Kluwer.

${ }^{11}$ STS 26 octubre 2012, no 642/2012 [CENDOJ: 28079110012012100608].

12 Vid. STS 485/2015 de 10 septiembre 2015 [ECLI: ES:TS:2015:3796]; STS 748/2014 de 11 diciembre 2014 [ECLI: ES:TS:2014:5099]; STS 200/2016 de 31 marzo 2016 [ECLI: ES:TS:2016:1419]; STS 664/2015 de 19 noviembre 2015 [ECLI: ES:TS:2015:492]; STS 230/2018 de 18 abril 2018 [ECLI: ES:TS:2018:1383].

${ }_{13}$ STS 20 octubre 2014, $\mathrm{n}^{\circ}$ 536/2014 [CENDOJ: 28079110012014100504]; STS 18 enero 2017, $\mathrm{n}^{\mathrm{o}}$ 18/2017 [CENDOJ: 28079110012017100002]. 
18. En el marco de las controversias que suscita el cambio de domicilio de los menores de edad cuando ya se han adoptado y están en vigor medidas judiciales relativas a la guarda y custodia del menor y al régimen de visitas a favor del progenitor no custodio, y como muy bien ha mostrado la profra. M. HERRANZ BALLESTEROS, son numerosas las resoluciones judiciales que han tenido que pronunciarse al respecto ${ }^{14}$.

19. El Reglamento $2201 / 2003$ recoge el concepto de "responsabilidad parental" y especifica que comprende "los derechos y obligaciones conferidas a una persona fisica o jurídica en virtud de una resolución judicial, por ministerio de la ley o por un acuerdo con efectos jurídicos, en relación con la persona o los bienes de un menor" (art.1.2, 1.3 y 2.7). Como señalan Alfonso-Luis Calvo Caravaca Y JAVIER CARRASCOSA GONZÁLEZ ${ }^{15}$, la responsabilidad parental es un concepto muy extenso que incluye entre otros: a) el derecho de custodia y el derecho de visita. El derecho de visita no se define por el Reglamento Bruselas II-bis. Se trata, en todo caso, de un concepto amplio y europeo. El Reglamento no excluye expresamente a los abuelos de la titularidad del derecho de visita.

20. Los trabajos preparatorios del Reglamento demuestran que la intención del legislador europeo fue no restringir el círculo de persona que pueden ser titulares del derecho de visita. Además, el Reglamento se aplica a todas las resoluciones en materia de responsabilidad parental y entre las mismas se incluye de modo inequívoco el derecho de visita. La interpretación amplia de este concepto garantiza la uniformidad de respuesta jurídica en la UE, la inexistencia de decisiones contradictorias y se garantiza el interés del menor, pues los tribunales y autoridades del Estado miembro de su residencia habitual decidirán en torno al derecho de visita sea cual fuere la persona que lo invoca.

21. Por todo ello, el concepto de derecho de visita debe comprender también el derecho de visita de los abuelos (STJUE 31 mayo 2018, C-335/17, Valcheva); b) La tutela, la curatela y otras instituciones análogas; c) la designación y las funciones de toda persona u organismo encargado de ocuparse de la persona o de los bienes del menor, de representarlo o de prestarle asistencia; d) El acogimiento del menor en una familia o en un establecimiento; f) Las medidas de Derecho Público, como la guarda de un menor o su acogimiento en centros públicos en caso de abandono, siempre que sean medidas que no afecten a su educación y a la salud. El concepto de responsabilidad parental incluye, la patria potestad regulada en el Derecho civil español.

22. La doctrina coincide mayoritariamente en que el legislador ha optado por no definir el concepto, toda vez que el mismo, por su carácter fáctico, debe ser establecido por el juzgador caso a caso, sin condicionamientos previos ${ }^{16}$.

23. Tal como acertadamente indicó la profra. P.Orejudo Prieto de Los Mozos ${ }^{17}$ el lugar en el que el menor tiene su residencia habitual es algo que puede resultar complejo determinar en numerosas

14 Vid. M. Herranz Ballesteros, "Análisis del consentimiento del progenitor al desplazamiento o la retención del menor en un estado distinto al de su residencia habitual. Su interpretación en el auto de la Audiencia Provincial Barcelona (Sección $18^{\mathrm{a}}$ ) de 27 de noviembre”, CDT, 11, 2, Octubre 2019, pp. 641-651; M. Herranz BALlesteros, “Aproximación al procedimiento prejudicial de urgencia a través de la protección internacional de menores”, en A. Cebrián SALVAt/I. Lorente Martínez (Dir.), Protección de menores y Derecho Internacional Privado, Granada, Editorial Comares, 2019, pp. 171-189; M. Herranz BALLESTEROS, "Traslado del domicilio del menor en la jurisprudencia del Tribunal Supremo", en A.-L. Calvo Caravaca/J. Carrascosa González (Dir.), El Tribunal Supremo y el Derecho Internacional Privado, vol. 2, Murcia, Rapid Centro Color S.L., 2019, pp. 567-586; M. Herranz Ballesteros, "La sustracción internacional de menores. A propósito de la STC 120/2002, de 20 mayo 2002, RDP, octubre 2002, pp. 754-770; ID., "El Reglamento 2201/2003. Alcance de la reforma en materia de sustracción internacional de menores en el espacio judicial europeo", Aranzadi Civil, nov.2004, pp. 13-31.

15 A.-L. Calvo Caravaca / J. Carrascosa González (Directores), Tratado de Derecho internacional privado, tomo. II, $18^{\mathrm{a}}$ edición, Tirant lo Blanch, Valencia, 2020, pp. 2029-2031.

16 Véase, al respecto, en la doctrina española a J. CARRASCOSA GonZÁLEZ, "Reglamento sucesorio europeo y actividad notarial", CDT, (Marzo 2014), Vol. 6, no 1, pp. 7.

17 P. Orejudo Prieto de los Mozos, "Diez años de aplicación e interpretación del Reglamento Bruselas II bis sobre crisis matrimoniales y responsabilidad parental (análisis de los aspectos de competencia judicial internacional)”, La Ley, Unión Europea, núm. 21, 2014, pp. 5-22. 
situaciones internacionales. Así, no es de extrañar que el TJUE se haya tenido que pronunciar sobre la interpretación del foro de la "residencia habitual del menor" en varias ocasiones. La interpretación del criterio no puede ser distinta en función de cuál sea la disposición aplicable ${ }^{18}$.

24. Dos pronunciamientos del TJUE, complementarios, y que analizan situaciones que pueden darse en los conflictos familiares han sido claves en la precisión del concepto de residencia habitual en el ámbito europeo: la primera, la STJUE de 28 de junio de $2018, H R^{19}$, resuelve un caso de un menor que, en el momento del conflicto, tenía claros vínculos con dos Estados miembro diferentes de la Unión (Bélgica y Polonia), tenía la nacionalidad de ambos y había residido alternativamente en los dos países. La segunda, la STJUE de 17 de octubre de $2018, U D^{20}$, resuelve un conflicto en el que se alegaba que la residencia habitual de una menor se encontraba en un Estado europeo en el que nunca había permanecido físicamente, introduciendo la madre en el proceso la alegación de estar sufriendo violencia de genero.

El TJUE vino a fijar así los criterios que deben tener en cuenta y cuáles no para establecer la residencia habitual del menor: Primero, la residencia del menor desde su nacimiento hasta separación con sus padres en un lugar concreto. Segundo, qué progenitor ejerce la guarda y custodia desde la separación, vinculado a su actividad profesional, más si se trata de una relación laboral por tiempo indefinido. Por último, el hecho de que el menor mantenga, en dicho lugar, un contacto regular con su otro progenitor, que sigue residiendo en él. Y no se deben tener en cuenta otros tres aspectos. Primero, las estancias aisladas y concretas, generalmente en periodos festivos, que el progenitor que ejerce en la práctica la guarda y custodia del menor ha efectuado, en el pasado, con el menor, en el territorio del Estado miembro de su origen. Segundo, los orígenes del progenitor de que se trata, relacionado con los vínculos de índole cultural del menor. Tercero, la eventual intención de dicho progenitor de establecerse con el menor, en el futuro, en ese mismo Estado miembro.

25. El legislador europeo ha seguido la misma línea en sus últimos instrumentos jurídicos, como en el Reglamento 1111/2019 ${ }^{21}$. Sin embargo, cabe defender que la concreción de la residencia hubiese sido positiva ${ }^{22}$, y en todo caso no en absoluto incompatible con la posibilidad de aportar una concreción de qué criterios tener en cuenta para ello según la Jurisprudencia del TJUE.

26. En efecto, el nuevo Reglamento (UE) 2019/1111 del Consejo de 25 de junio de 2019 relativo a la competencia, el reconocimiento y la ejecución de resoluciones en materia matrimonial y de responsabilidad parental, y sobre la sustracción internacional de menores que sustituirá al Reglamento 2201/2003, no modifica el enfoque descrito en relación a la residencia habitual, aunque sí introduce criterios hermenéuticos de gran interés en sus Considerandos, cuando, por ejemplo en el $n^{0} 20$ subraya que para salvaguardar el interés superior del menor la competencia debe en primer lugar determinarse con arreglo al criterio de proximidad. Y con arreglo a éste señala que son los órganos jurisdiccionales del Estado miembro en el cual el menor tiene su residencia habitual los que deben ser competentes, excepto en ciertas situaciones contempladas en el propio Reglamento, por ejemplo cuando se produce un cambio de residencia del menor o en caso de acuerdo entre los titulares de la responsabilidad parental.

\footnotetext{
${ }^{18}$ En concreto, advierte el TJUE que el concepto de la residencia habitual del menor de los arts. 2.11 y 11 RBII bis no puede diferir en su contenido del que se retiene en la jurisprudencia anterior, relativa a los arts. 8 y 10 RBII bis: vid. § 54 STJUE de 9 de octubre de 2014, Asunto C-376/14, C c M.

19 Asunto C-512/17, ECLI:EU: C: 2018: 513.

20 Asunto C-393/18, ECLI: EU:C:2018:835.

${ }^{21}$ Reglamento 1111/2019 de 25 de junio de 2019, DOUE L178/1 de 2 de julio de 2019, del texto refundido de Bruselas II bis. Incluye en su artículo 2 una única definición novedosa, la de menor como toda persona que tenga menos de 18 años. La residencia habitual nunca se definió ni el informe inicial de la Comisión de 15 de abril de 2014 ni en la posición del Parlamento de 28 de abril de 2016. Tampoco en la propuesta de la Comisión de 30 de junio de 2016, o el Informe del Parlamento de 21 de noviembre de 2017.

${ }^{22}$ Misma opinión se puede observar en Gandía Sellens, M.A., "La responsabilidad parental y la sustracción de menores en la propuesta de la Comisión para modificar el RB II Bis: algunos avances, retrocesos y ausencias", AEDIPr, T. XVII, 2017 , pp. 799 a 820 , p. 814.
} 
27. El propio Reglamento señala que cuando no haya aún procedimientos en curso en materia de responsabilidad parental y la residencia habitual del menor cambie a raíz de un traslado lícito del menor, la competencia debe seguirle con el fin de mantener la proximidad. Y en particular, en una previsión que contempla exactamente el caso resuelto en la decisión objeto del presente comentario, señala que para los procedimientos que ya estén en curso, la seguridad jurídica y la eficiencia de la justicia justifican el mantenimiento de la competencia hasta que los procedimientos hayan desembocado en una resolución definitiva o hayan concluido de otra forma.

\section{Atribución competencial frente a distribución: competencia judicial internacional versus com- petencia territorial interna}

28. La competencia judicial internacional se articula a partir de las normas (de fuente europea, convencional e interna) que integran nuestro sistema de Derecho internacional. Aplicar para esa categoría de competencia judicial internacional una norma de competencia interna supone una merma de la tutela judicial efectiva. En esta línea se ha manifestado el Tribunal Constitucional. En su sentencia $61 / 00^{23}$ afirma que tal solución supone una vulneración del derecho a la tutela judicial efectiva consagrado en el artículo $24 C E$ en la medida en que impide el conocimiento del asunto por cualquier tribunal español basándose para ello en una regla que únicamente reparte la competencia entre los tribunales españoles y cuyos principios y objetivos difieren de las disposiciones que ordenan la competencia judicial internacional de los órganos judiciales españoles. Las normas de competencia judicial internacional delimitan el ejercicio de la potestad jurisdiccional atendiendo a las exigencias derivadas del derecho a la tutela judicial efectiva proyectada en el principio de proximidad. Solo en ellas puede darse respuesta a la cuestión de si es posible que los tribunales españoles conozcan de una determinada pretensión, pues solo ellas responden a las exigencias necesarias para que nuestros tribunales puedan asumir la tutela judicial efectiva del caso concreto ${ }^{24}$.

29. En el caso resuelto por la decisión judicial objeto del presente comentario cabe deducir que no existe aparentemente un debate o cuestión acerca de la competencia judicial internacional de los tribunales españoles, teniendo en cuenta que lo que se plantea en el recurso de apelación es una declinatoria por falta de competencia territorial del Juzgado de Primera Instancia $\mathrm{n}^{\circ} 3$ de Córdoba. La Audiencia Provincial señala que además de que la recurrente validó la competencia de los tribunales españoles, ésta recaía sobre éstos al amparo del artículo 8 del Reglamento Bruselas II bis porque el momento en el que hay que fijar y concretar el foro de la residencia habitual de los menores es el momento procesal de la presentación de la demanda. $Y$ en el caso ahora planteado, en ese momento procesal su residencia habitual era España.

\section{Consideraciones finales}

30. Por todo lo expuesto es razonable concluir que la decisión judicial comentada acierta, es correcta cuando señala que la competencia le corresponderá al Juez que fuera competente en atención al foro o criterio correspondiente a la residencia habitual de los menores al tiempo de la presentación de la demanda, no al tiempo en que se dictó la resolución apelada y que a su vez, la recurrente, una vez que planteó la cuestión de competencia ante el Juzgado de Primera Instancia $\mathrm{n}^{\mathrm{0}} 3$ de Córdoba que inicialmente conoció del asunto, hubo de haber instado la falta de competencia judicial internacional de los Tribunales españoles ya en ese momento, sin que se pueda reservar esa cuestión para posterior momento por preclusión de esa posibilidad.

\footnotetext{
${ }^{23}$ Sentencia del Tribunal Constitucional 61/00, de 13 de marzo de 2000 (BOE, $\mathrm{n}^{\circ} 90$ de 20 de abril).

${ }^{24}$ M. SABido RodríGuez, "algunas cuestiones que plantea la modificación, en España, de las medidas de guarda y custodia adoptadas por tribunales extranjeros a la luz del auto de la audiencia provincial de Barcelona de 29 de enero de 2020", CDT, (Marzo 2021), Vol. 13, No 1, pp. 1085-1094.
} 
31. Desde nuestra dimensión de análisis internacional privatista debe tenerse presente que el objeto de la litis planteada en el recurso de apelación ante la Audiencia de Córdoba es la falta de competencia territorial del Juzgado de Primera Instancia $n^{0} 3$ de Córdoba; de ello cabe inferir que en realidad no existe a priori un debate o cuestión acerca de la competencia judicial internacional de los tribunales españoles. Solo cuando esta premisa (competencia de atribución, determinada en este caso por el Reglamento Bruselas II bis) deviene indiscutida podrá abrirse el debate sobre la competencia de distribución interna española (territorial y/o funcional). Pero cuando, como ocurre en este caso, el debate procesal se instala en esta segunda dimensión procesal interna o de distribución no existe en puridad una problemática procesal internacional privatista.

32. Las circunstancias procesales y fácticas del supuesto analizado en la decisión judicial comentada conducen sin duda alguna a conferir la competencia judicial internacional a los tribunales españoles: en el transcurso de un procedimiento judicial ya iniciado los menores cambian de residencia de manera legal (con consentimiento del padre); en este supuesto, y dado que el juez del Estado (en este caso, España) de la primera residencia habitual no se hubiese pronunciado aún, es decir, si no se hubiera dictado sentencia, dicho órgano judicial continuará reteniendo su competencia aunque el menor cambie su residencia en ese ínterin procesal. Y todo ello, siguiendo el principio general de perpetuatio fori, principio que se aplica en el marco del Reglamento 2201/2003, atendiendo a las previsiones de su art.8. 